\title{
Characterization of a Naturally Occurring Mutation (Arg-460 to Gly) Close to FAD in Human Dihydrolipoamide Dehydrogenase
}

\author{
Lin Yuan and Hakjung Kim* \\ Department of Chemistry and College of Natural Science, Daegu University, Kyoungsan 712-714, Korea \\ *E-mail: hjkim@daegu.ac.kr \\ Received September 6, 2010, Accepted September 30, 2010
}

Key Words: Dihydrolipoamide dehydrogenase, Pyridine nucleotide-disulfide oxidoreductase, Site-directed mutagenesis, Dihydrolipoamide deficiency, $\alpha$-keto acid dehydrogenase complexes

Dihydrolipoamide dehydrogenase (E3) (dihydrolipoamide: $\mathrm{NAD}^{+}$oxidoreductase; EC 1.8.1.4) is present as a common component of flavoproteins in pyruvate, $\alpha$-ketoglutarate and branched-chain $\alpha$-keto acid dehydrogenase complexes ${ }^{1}$ and in the glycine cleavage system. ${ }^{2}$ It catalyzes the reoxidation of the dihydrolipoyl groups of acyltransferase components of $\alpha$-keto acid dehydrogenase complexes and the hydrogen-carrying proteins of the glycine cleavage system. It is a homodimeric flavoenzyme with each subunit containing one FAD. ${ }^{3}$ The clinical symptoms of E3 deficiency range from severe neurological defects, such as Leigh syndrome, to less severe exertional fatigue in childhood. A reduction of E3 activity results in decreases of activities of all three of the above complexes as E3 is a common component. One mutation found in an infant girl with E3 deficiency is the substitution of Arg-460 to Gly. ${ }^{4}$ She was apparently normal at birth but had transient neonatal hypoglycemia and poor sucking. She had a history of developmental delay and hypotonia, associated with metabolic acidosis. She had elevated blood lactate, pyruvate, and plasma branched-chain amino acids.

Site-directed mutagenesis has been used for the structurefunction study of human E3. ${ }^{5-7}$ By the site-specific mutation of Arg-460 to Gly, the effects of this naturally occurring mutation in the structure and function of human E3 were examined. The site-directed mutagenesis was performed using a mutagenesis kit (iNtRON Biotechnology, Sungnam, ROK). Two mutagenic primers were used for the mutations. Primer A (5'-CAAGATT TGCTTCTCCAAAAGCCTCTGATAAGG-3': with mismatched bases underlined) is an anti-sense oligomer with point mutations to convert Arg-460 (AGA) to Gly (GGA). Primer B (5'-CCTTATCAGAGGCTTTTGGAG AAGCAAATCTTG-3': with mismatched bases underlined) is the corresponding sense oligomer of primer A. Polymerase chain reactions (PCR) were performed in a programmable PCR machine using the human E3 expression vector $\mathrm{pPROEX}-1$ :E3 as a template. Mutations were confirmed by DNA sequencing.

The purification of the Gly-460 mutant was performed using a nickel affinity column as described in elsewhere. ${ }^{6} \mathrm{E} 3$ assay was performed at $37^{\circ} \mathrm{C}$ in a $50 \mathrm{mM}$ potassium phosphate buffer (pH 8.0) containing 1.5 mM EDTA with variable concentrations of the substrates, dihydrolipoamide and $\mathrm{NAD}^{+}$. The activity was recorded spectrophotometrically by observing the reduction of $\mathrm{NAD}^{+}$at $340 \mathrm{~nm}$. One unit of activity was defined as $1 \mu \mathrm{mol}$ of $\mathrm{NAD}^{+}$reduced per min. E3 activity data were applied to the SigmaPlot Enzyme Kinetics Module program (Systat Software Inc., San Jose, USA). The program generated double reciprocal plots as shown in Figure 1 and calculated kinetic parameters. Kinetic experiments were performed in triplicate. $k_{\text {cat }}$ was determined as $264+4 \mathrm{~s}^{-1}$ which was about $3.4 \times$ lower than the $899+114 \mathrm{~s}^{-1}$ of normal human E3, indicating that the mutation severely affected the catalytic process of the conversion of the substrates to products. The $K_{m}$ value for dihydrolipoamide was determined as $0.12+0.01 \mathrm{mM}$, approximately $5.3 \times$ lower than the $0.64+0.06 \mathrm{mM}$ of normal human E3, indicating that the mutation makes the enzyme binding more efficient to dihydrolipoamide. However, the $K_{m}$ value for NAD ${ }^{+}$was determined as $0.26+0.01 \mathrm{mM}$, approximately $1.4 \times$ larger than the $0.19 \pm 0.02 \mathrm{mM}$ of normal human E3, indicating that the mutation makes the enzyme binding less efficient to $\mathrm{NAD}^{+}$.

To examine whether structural changes occur in the Gly-460 mutant, UV-visible absorption and fluorescence spectroscopies were performed. Due to the prosthetic group FAD, human E3 has the characteristic UV-visible absorption spectrum of flavoproteins shown in Figure 2A. The overall shape of the mutant spectrum (solid line) was similar to that of the wild-type human E3 spectrum (dotted line). However, clear evidence of structural changes in the mutant came from the fluorescence study. When enzymes were excited at $296 \mathrm{~nm}$, two fluorescence emissions were observed for both mutant and wild-type E3s, as shown in Figure 2B. The first emission from $305 \mathrm{~nm}$ to $400 \mathrm{~nm}$ was mainly
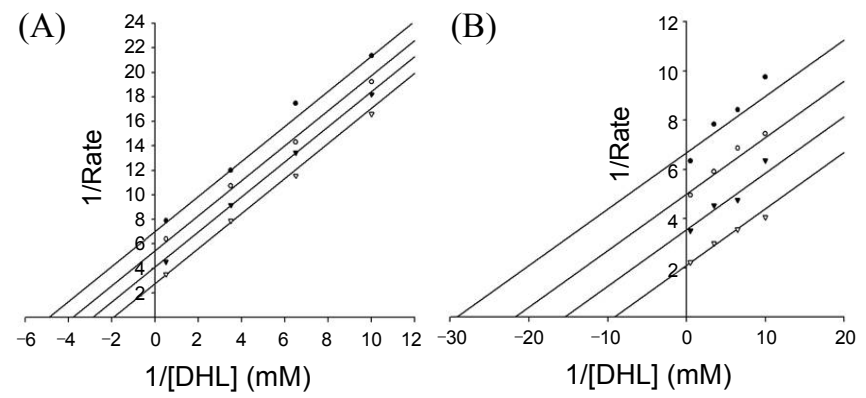

Figure 1. Double reciprocal plots for the wild-type (A) and mutant (B) human E3s. E3 activities were determined at $37^{\circ} \mathrm{C}$ in a $50 \mathrm{mM}$ potassium phosphate buffer ( $\mathrm{pH}$ 8.0) containing 1.5 mM EDTA with variable concentrations of the substrates, dihydrolipoamide (DHL) and $\mathrm{NAD}^{+}$. Plots were drawn with the SigmaPlot Enzyme Kinetics Module program. The $\mathrm{NAD}^{+}$concentrations from top to bottom are $0.1,0.154$, 0.286 and $2 \mathrm{mM}$. The DHL concentrations from right to left are 0.1 , $0.154,0.286$ and $2 \mathrm{mM}$. 

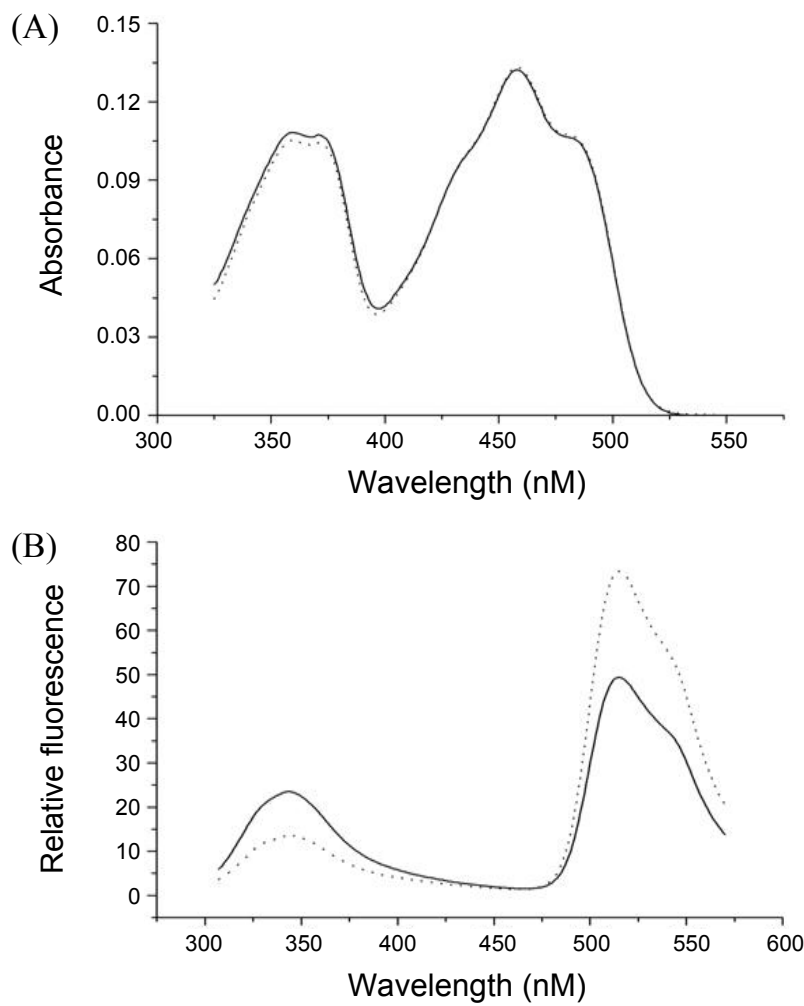

Figure 2. Spectroscopic properties of both mutant and wild-type human E3s. A; UV-visible spectra of the mutant (solid line) and wild-type (dotted line) recombinant human E3s. The spectra were recorded using a SPECORD200 spectrophotometer and the data from $325 \mathrm{~nm}$ to 550 $\mathrm{nm}$ were transferred to an ASCII file. The spectra were then drawn using the MicroCal Origin program. B; Fluorescence spectra of the mutant (solid line) and wild-type (dotted line) recombinant human E3s. Enzymes were excited at $296 \mathrm{~nm}$ and the emissions were observed from $305 \mathrm{~nm}$ to $575 \mathrm{~nm}$. The data were transferred to an ASCII file and the spectra were then drawn using the MicroCal Origin program.

due to Trp. The second emission from $480 \mathrm{~nm}$ to over $550 \mathrm{~nm}$ was attributable to FAD. In human E3, the Trp fluorescence was quenched by energy transfer from Trp to FAD. When the fluorescence spectra of E3s were compared, a noticeable difference was found in the ratio between the relative intensities of the first and second fluorescence emissions. The ratio (about 2.1) between relative intensities of the first and second fluorescence emissions of the mutant (solid line) was lower than the $\sim 5.4$ of the wild-type enzyme (dotted line). This indicates that the energy transfer from Trp to FAD was disturbed in the Gly-460 mutant. In human E3, Arg-460 is located close to the prosthetic group FAD as shown in Figure 3. The side chain of Arg-460 can form several H-bonds with back bone CO groups of Val-448, Thr-454 and Ala-446. The mutation of Arg-460 to Gly, having a hydrogen atom as a side chain, would prevent these interactions. The mutation would give a vacancy of $113.3 \AA^{3}$ at the residue- 460 . The mutation also removes the positively charged Arg side chain. This could cause structural changes that alter the kinetic properties of the mutant. The structural changes brought about by the mutation of Arg-460 to Gly could affect the structure of human E3 so that the energy transfer from Trp residue(s) to FAD was interfered with.

In this study, the effects of a naturally occurring mutation,

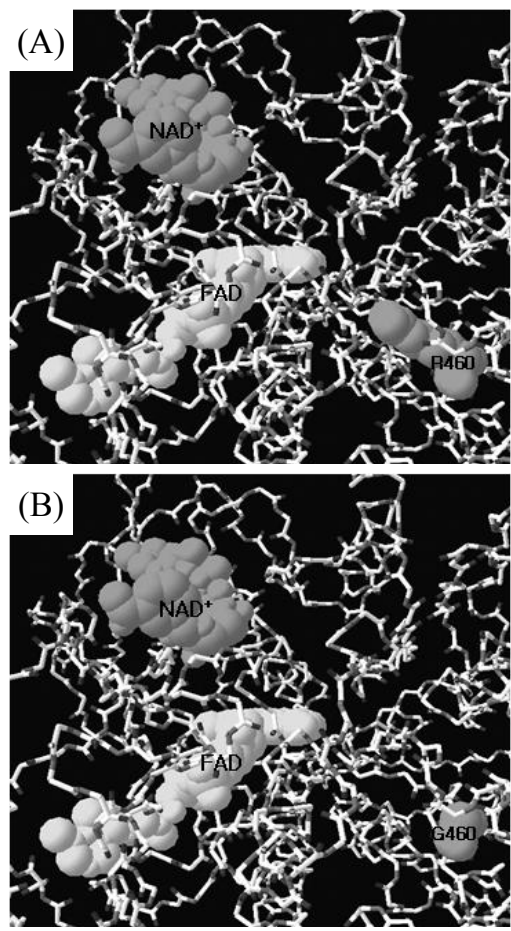

Figure 3. Location of Arg-460 in human E3. The FAD, NAD ${ }^{+}$and mutated residues are shown in space-filled structures and other residues are shown in backbone structures. A; Before the mutations. B; After the mutations (Arg-460 to Gly). The PDB code for the human E3 structure is $1 \mathrm{ZMC}$.

(Arg-460 to Gly) close to FAD, on the structure and function of human E3 were examined using site-directed mutagenesis, E3 activity measurement and spectroscopic methods. The results show that the E3 deficiency of the patient mainly resulted from a $k_{\text {cat }}$ reduction of $3.4 \times$ due to the mutation of Arg-460 to Gly. The mutation could cause structural changes which interfere with fluorescence resonance energy transfer from Trp residue(s) to FAD. These findings show that the conservation of Arg460 residue in human E3 is very important to its structure and function.

Acknowledgments. The authors thank Dr. Mulchand S. Patel (University at Buffalo, the State University of New York) for the generous gift of an $E$. coli XL1-Blue containing a human E3 expression vector. The authors are grateful to Dr. Tai Jong Kang (Daegu University) for providing a fluorometer. This research was supported in part by the Daegu University Research Grant, 2009.

\section{References}

1. Reed, L. J. Acc. Chem. Res. 1974, 7, 40.

2. Walker, J. L.; Oliver, D. J. J. Biol. Chem. 1986, 261, 2214.

3. Brautigam, C. A.; Chuang, J. L.; Tomchick, D. R.; Machius, M.; Chuang, D. T. J. Mol. Biol. 2005, 350, 543.

4. Hong, Y. S.; Kerr, D. S.; Craigen, W. J.; Tan, J.; Pan, Y.; Lusk, M.; Patel, M. S. Human. Mol. Genet. 1996, 5, 1925.

5. Kim, H.; Patel, M. S. J. Biol. Chem. 1992, 267, 5128.

6. Yuan, L.; Cho, Y.-J.; Kim, H. Bull. Korean Chem. Soc. 2009, 30, 777.

7. Yuan, L.; Cho, Y.-J.; Kim., H. Bull. Korean Chem. Soc. 2008, 29, 2327. 